\title{
Publisher Correction: Environmental eustress modulates $\beta$-ARs/CCL2 axis to induce anti-tumor immunity and sensitize immunotherapy against liver cancer in mice
}

Chaobao Liu (1), Yang Yang, Cheng Chen (1), Ling Li, Jingquan Li, Xiaonan Wang, Qiao Chu, Lin Qiu, Qian Ba (1), Xiaoguang Li (1) \& Hui Wang

Correction to: Nature Communications https://doi.org/10.1038/s41467-021-25967-9, published online 30 September 2021.

In this article Jingquan $\mathrm{Li}$ was incorrectly denoted as being one of the equally contributing authors. The original article has been corrected. Following the publication of the original article, it was noted that, due to a typesetting error, the figure labelling for Figure 6A-G was incorrect in the Results section "EE overcomes PD-L1 based checkpoint blockade resistance". The PDF and HTML versions of the Article have been corrected.

Published online: 14 October 2021

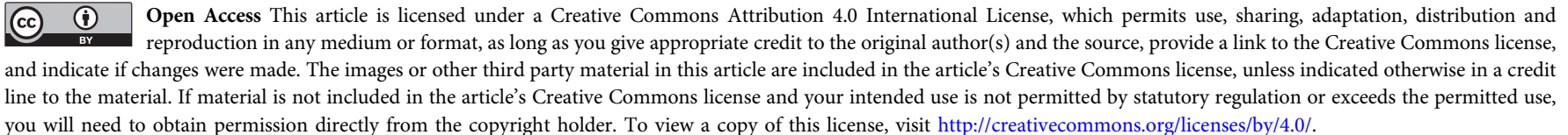

(c) The Author(s) 2021 